\title{
Estudio comparativo de la variación de las propiedades hídricas y el aspecto de la piedra natural y el ladrillo tras la aplicación de 4 tipos de anti-grafiti
}

\section{Comparative study of the variation of the hydric properties and aspect of natural stone and brick after the application of 4 types of anti-graffiti}

\author{
O. García(*), I. Rz-Maribona(*), A. Gardei(**), M. Riedl(**), Y. Vanhellemont(***), M. L. Santarelli(****), \\ J. Strupi Suput $(* * * * *)$
}

Recepción/Received: 28-XII-07

Aceptación/Accepted: 28-XI-08

Publicado online/Online publishing: 9-II-10

\section{RESUMEN}

En este artículo se presenta un estudio comparativo del comportamiento de diferentes tipos de anti-graffiti comerciales sobre piedra natural y ladrillo. Para ello se seleccionaron 8 tipos de sustratos porosos de diferentes países europeos, sobre los que se aplicaron 4 anti-graffiti de distinta naturaleza química. Posteriormente se estudiaron las variaciones en sus propiedades hídricas y de aspecto (color y brillo) con respecto a los sustratos no tratados, en el laboratorio.

Los resultados obtenidos han permitido evaluar la idoneidad de 4 de los principales tipos de formulaciones químicas más frecuentemente utilizadas como anti-graffiti sobre sustratos porosos. El estudio concluye que el antigraffiti de sacrificio de composición parafínica es el producto que reduce en menor medida las propiedades hídricas de los sustratos porosos estudiados, y que menores cambios de color produce en los mismos.

Palabras clave: sustratos porosos, tratamientos superficiales, recubrimientos anti-grafiti; propiedades hídricas; color; brillo.

\section{SUMMARY}

In this article a comparative study of the behaviour of different commercial anti-graffiti on natural stone and brick is presented. 8 different European substrates were selected and 4 commercial anti-graffiti of different chemical nature were applied on these substrates. The variations of their hydric properties and aspect (colour and gloss) with regard to the untreated substrates were later studied in the laboratory.

The results obtained permitted to assess the suitability of 4 of the main types of chemical formulations employed to be used as anti-graffiti. This study concludes that the sacrificial anti-graffiti with polymeric paraffins in its composition presents the lowest reductions of the hydric properties of the studied substrates, being also the variations in colour the least perceptible.

Keywords: porous substrates, surface treatments, antigraffiti coatings; hydric properties; colour; gloss.

(*) Labein-Tecnalia (Derio, España).

(**) BAM (Berlín, Alemania).

$(* * *)$ BBRI (Bruselas, Bélgica)

$(* * * *)$ CISTeC, Università di Roma La Sapienza (Roma, Italia).

$(* * * * *)$ ZAG (Ljubljana, Eslovenia). 


\section{INTRODUCCIÓN}

Los grafiti constituyen una seria amenaza para los monumentos y edificios históricos. Este fenómeno social surgido hace ya 40 años (1), además de no contar con buena reputación social, perjudica en la mayoría de los casos a los materiales que hoy en día encontramos en nuestro Patrimonio construido. Dado que los materiales presentes en estos edificios son frecuentemente de naturaleza porosa (piedra natural, ladrillo, mortero), la penetración de los pigmentos suele ser profunda. Por si esto fuera poco, los métodos y productos utilizados en la limpieza de grafiti no son en muchos casos los más adecuados, por lo que los resultados suelen ser insatisfactorios.

Con el fin de proteger el sustrato del grafiti, puede aplicarse un recubrimiento anti-grafiti, el cual facilita posteriormente la limpieza de la pintada. Existen dos grandes familias de anti-grafiti (2): los anti-grafiti permanentes y los de sacrificio. Los primeros son productos que no son eliminados durante el proceso de limpieza de grafiti y pueden aguantar hasta más de 15 procesos de limpieza. Están diseñados principalmente para materiales de baja porosidad, tales como los metales o el vidrio. En su composición química presentan, poliuretanos y resinas epo$x i$, los cuales suelen formar una capa impermeable al agua sobre el sustrato tratado, por lo que su uso en los sustratos históricos no está recomendado (3-5). En el caso de segundo tipo, el de los anti-grafiti de sacrificio $(6,7)$, el producto anti-grafiti es eliminado junto con el grafiti, por lo que el producto protector debe ser aplicado de nuevo. Este tipo de anti-grafiti suele ser más adecuado para la conservación del patrimonio, pues reducen en menor medida las propiedades hídricas de los sustratos, sobre todo la permeabilidad al vapor de agua, aunque pueden resultar también inadecuados, debido al tratamiento de limpieza que algunos de ellos llevan asociado (agua caliente a alta presión). Son productos de base cerosa, polisacáridos y acrilatos.

Finalmente, existen también los productos anti-grafiti "semi-permanentes", los cuales no son eliminados durante el proceso de limpieza de grafiti, pero presentan una durabilidad menor que los anti-grafiti permanentes (2-3 limpiezas). Pueden ser de una sola capa o multicapas $y$, composicionalmente, pertenecen fundamentalmente a la familia de los siloxanos $(8,9)$.

El objeto de este trabajo es la evaluación del efecto que producen 4 de los principales tipos de anti-grafiti comerciales sobre diferentes tipos de sustratos porosos representativos del Patrimonio Cultural de diversos países europeos, con el fin de determinar su idoneidad para la protección de los materiales históricos mencionados.

\section{INTRODUCTION}

Graffiti are a serious menace for historical monuments and buildings. This social phenomenon that emerged 40 years ago (1) not only has a negative social reputation but also damages in most of the cases the materials found today in our Built Heritage. Considering the materials present in such constructions are porous (natural stone, brick, mortar), the penetration of the pigments is commonly high. Additionally, the methods and products used in the cleaning of graffiti are not very frequently the most suitable. As a consequence of this, results are often unsatisfactory.

With the aim of protecting the substrate from graffiti an anti-graffiti coating can be applied and this facilitates the subsequent cleaning of graffiti. There two main anti-graffiti families (2): permanent and sacrificial. The first type of products is not eliminated during the removal of graffiti and can withstand in some cases more than 15 cleaning cycles. They are principally designed to be applied on low porosity materials such as metals or glass. They are based on polyurethanes and epoxy resins, and they work by forming a film on the treated substrate which is impermeable to water, thus their use on historical substrates is not recommended (3-5). In the case of the second type of coatings, sacrificial anti-graffiti (6, $7)$, the protective product is removed together with the graffiti, therefore it must be reapplied just after cleaning. This type of products is more suitable for Cultural Heritage because they reduce to a lesser extent the hydric properties of the porous substrates, especially the water vapour permeability. However, they can also be inadequate for historical materials due to the cleaning methods normally used (highpressure hot water). These products are based on waxes, polysaccharides and acrylates.

Finally, there are also the "semi-permanent" antigraffiti products, which are not eliminated during the cleaning process of graffiti, but present a lower durability than permanent anti-graffiti (2-3 cleanings). They can be single-coating or multicoating and compositionally they mainly belong to the family of siloxanes $(8,9)$.

The main purpose of this work is the evaluation of the effect produced by the application of 4 of the main commercial anti-graffiti types on different porous substrates considered to be representative of the Cultural Heritage of different European countries, in order to determine the suitability of such products for the protection of the mentioned historical materials. 


\section{PARTE EXPERIMENTAL}

\subsection{Materiales}

Para la realización de este estudio se han empleado 4 tipos de anti-grafiti comerciales*, que corresponden a los siguientes tipos de formulaciones:

- ANTI-GRAFITI 1: copolímero de acrilato.

- ANTI-GRAFITI 2: emulsión acuosa de polímeros parafínicos.

- ANTI-GRAFITI 3: poliuretano.

- ANTI-GRAFITI 4: etil metacrilato

La selección de estos 4 tipos de formulaciones se ha efectuado en función de los siguientes criterios (Tabla 1):

- Permanencia y reversibilidad: anti-grafiti permanente y de sacrificio (se han seleccionado de ambos tipos).

- Familia de producto (composición química).

- Frecuencia de utilización en sustratos porosos. Experiencia de utilización por parte de los investigadores participantes en el estudio.

- Idoneidad para su utilización en sustratos porosos, según ficha comercial.

Se escogieron productos anti-grafiti de base química diferente, siendo uno de ellos no recomendado por el fabricante en la protección de sustratos minerales porosos (caso del anti-grafiti 3), con el fin de estudiar las diferencias de la aplicación de productos de comportamiento muy diferente.

\section{EXPERIMENTAL SECTION}

\subsection{Materials}

4 types of commercial anti-graffiti were selected for the performance of this study. These products correspond to different chemical formulations*:

- ANTI-GRAFFITI 1: acrylate copolymer.

- ANTI-GRAFFITI 2: aqueous emulsion of paraffin polymers.

- ANTI-GRAFFITI 3: polyurethane.

- ANTI-GRAFFITI 4: ethyl methacrylate.

The selection of these 4 types of formulations was performed according to the following criteria (Table 1):

- Permanence and reversibility: permanent anti-graffiti and sacrificial anti-graffiti (both types of anti-graffiti were selected).

- Product family (chemical composition).

- Frequency of use in porous substrates. Previous experience of use by the researchers participating in the study.

- Suitability for use on porous substrates, according to technical data sheet.

In order to study the differences of the application of products presenting a very diverse behaviour, it was decided to choose anti-graffiti products of very different chemical composition, being one of the products not recommended by the manufacturer in the protection of porous mineral substrates (i.e. anti-graffiti 3).

Tabla 1 / Table 1

Tipos de anti-grafiti estudiados y criterios de elección. Studied anti-graffiti types and selection criteria.

\begin{tabular}{|c|c|c|c|}
\hline Anti-grafiti & Familia / Family & $\begin{array}{c}\text { Frecuencia de utilización en sustratos } \\
\text { porosos / } \\
\text { Frequency of use in porous } \\
\text { substrates }\end{array}$ & $\begin{array}{c}\text { Idoneidad para su utilización en } \\
\text { sustratos porosos/ } \\
\text { Suitability for its use in porous } \\
\text { substrates }\end{array}$ \\
\hline Anti-grafiti 1 & $\begin{array}{c}\text { Copolímero de acrilato } \\
\text { Acrylate copolymer }\end{array}$ & Media / Medium & Media-alta / Medium-high \\
\hline Anti-grafiti 2 & $\begin{array}{c}\text { Polímeros parafínicos } \\
\text { Paraffin polymers }\end{array}$ & Alta / High & Alta / High \\
\hline Anti-grafiti 3 & $\begin{array}{c}\text { Poliuretano } \\
\text { Polyurethane }\end{array}$ & Muy baja / Very low & Muja / Very low \\
\hline Anti-grafiti 4 & Ethyl methacrilato & Media / Medium & Alta / High \\
\hline
\end{tabular}

Estos 4 productos fueron aplicados sobre 8 tipos de sustratos porosos, los cuales se seleccionaron atendiendo a los siguientes criterios:

* No se proporcionan los nombres comerciales de los anti-grafiti utilizados al objeto de preservar la confidencialidad de los fabricantes y suministradores.
These 4 products were applied on 8 different porous substrates, which were selected following the criteria defined below:

* Commercial names have been omitted in order to preserve the confidenciality of the industrial manufacturers 
- Distribución geográfica en Europa.

- Tipo de material (arenisca, caliza, travertino, ladrillo).

- Porosidad y tamaño de poro.

La Tabla 2 muestra los 8 tipos de sustrato seleccionados, así como los criterios empleados para esta selección. La porosidad y el tamaño de poro se midieron empleando un porosímetro de mercurio Carlo Erba 2000.
- Geographical distribution throughout Europe.

- Type of material (sandstone, limestone, travertine, brick).

- Porosity and pore size.

Table 2 shows the 8 selected substrates, as well as the criteria used for their selection. The porosity and pore size were measured by using a Carlo Erba 2000 mercury porosimeter.

Tabla 2 / Table 2

Sustratos estudiados.

Studied substrates.

\begin{tabular}{|c|c|c|c|c|c|c|}
\hline \multirow{2}{*}{ Sustrato / Substrate } & \multirow{2}{*}{$\begin{array}{l}\text { Identificación / } \\
\text { Identification }\end{array}$} & \multirow{2}{*}{$\begin{array}{l}\text { País / } \\
\text { Country }\end{array}$} & \multicolumn{2}{|c|}{$\begin{array}{l}\text { Porosidad (\%) / } \\
\text { Porosity (\%) }\end{array}$} & \multicolumn{2}{|c|}{$\begin{array}{c}\text { Tamaño de poro }(\mu \mathrm{m}) / \\
\text { Pore size }(\mu \mathrm{m})\end{array}$} \\
\hline & & & $\begin{array}{l}\text { Valor medio / } \\
\text { Average value }\end{array}$ & $\sigma$ & $\begin{array}{l}\text { Valor medio / } \\
\text { Average value }\end{array}$ & $\sigma$ \\
\hline $\begin{array}{l}\text { Arenisca silícea de Villamayor / } \\
\text { Villamayor siliceous sandstone }\end{array}$ & VILLA & España / Spain & 15.1 & 3.2 & 2.4 & 0.3 \\
\hline $\begin{array}{l}\text { Arenisca silícea de Galdakao / } \\
\text { Galdakao siliceous sandstone }\end{array}$ & GALD & España / Spain & 15.8 & 0.1 & 1.3 & 0.2 \\
\hline $\begin{array}{l}\text { Arenisca calcárea de Strtenica / } \\
\text { Strtenica calcareous sandstone }\end{array}$ & STRT & Eslovenia/ Slovenia & 15.8 & 1.5 & 1.7 & 0.4 \\
\hline $\begin{array}{l}\text { Arenisca calcárea de Baumberg / } \\
\text { Baumberg calcareous sandstone }\end{array}$ & BAUM & $\begin{array}{l}\text { Alemania / } \\
\text { Germany }\end{array}$ & 21.1 & 1.5 & 0.5 & 0.03 \\
\hline Caliza azul belga / Belgian blue limestones & BLUE & Bélgica / Belgium & 1.4 & 0.4 & 0.004 & $<0.001$ \\
\hline Caliza de Balegem / Balegem limestones & BALE & Bélgica / Belgium & 6.2 & 1.1 & 0.004 & $<0.001$ \\
\hline Travertino romano / Roman travertine & TRAV & Italia / Italy & 2.5 & 1.2 & 2.4 & 3.4 \\
\hline Ladrillo / Brick & LADR & $\begin{array}{l}\text { Alemania / } \\
\text { Germany }\end{array}$ & 19.5 & 0.1 & 3.1 & 0.3 \\
\hline
\end{tabular}

o : Desviación estándar / Standard deviation.

Con el fin de comparar las características de los materiales tratados (con anti-grafiti) con las del material no tratado (sin anti-grafiti), en todos los ensayos se incluyeron probetas patrón (sin recubrimiento).

\subsection{Preparación de muestras}

Las muestras de piedra y ladrillo utilizadas fueron suministradas por canteros y fabricantes de materiales cerámicos, por lo que estas muestras no habían sido sometidas a procesos de deterioro aparentes. La geometría y tamaño de estas muestras fue, en cada caso, la establecida por el método de ensayo normalizado a utilizar, que se presenta en el siguiente apartado.

Previamente a la aplicación de los productos anti-grafiti, las muestras se acondicionaron en el laboratorio a $23 \pm$ $2{ }^{\circ} \mathrm{C}$ y $50 \pm 5 \%$ r.h. durante al menos 7 días. La aplicación del anti-grafiti se efectuó mediante un rodillo pequeño de pintar de fibra corta en una sola de las caras de las muestras. Para controlar la cantidad de producto aplicada, cada probeta, así como el rodillo, se pesaron antes y después de aplicar el producto. Después de
Blank specimens (untreated samples) were used in all the tests with the aim of comparing the properties of the treated materials (with anti-graffiti) with the untreated materials (without anti-graffiti).

\subsection{Sample preparation}

The samples of natural stone and brick were supplied by different quarries and manufacturers of ceramic materials, thus the samples were not exposed to apparent natural weathering processes. The geometry and size of the specimens were established according to the specifications given in the normalized procedures, which are defined in the next section.

Before testing, samples were conditioned in the laboratory at $23 \pm 2{ }^{\circ} \mathrm{C}$ and $50 \pm 5 \%$ r.h. during at least 7 days prior to the product application. The anti-graffiti application was performed with a small, short-fibred paint roll in one of the sides of the tested samples. In order to control the applied anti-graffiti amount, each specimen and the paint roll were weighted before and after the product application. After weighing the paint-roll, the anti-graffiti product was applied 
pesar el rodillo, se aplicó el producto en la superficie del sustrato poroso, hasta conseguir una distribución homogénea del anti-grafiti.

El número de capas de anti-grafiti a aplicar, así como el periodo de secado entre capa y capa se estableció de acuerdo con las recomendaciones proporcionadas en la ficha técnica del producto. En la Tabla 3 se indica el número de capas aplicadas en cada uno de los casos: on the surface of the porous substrate until a uniform distribution of the anti-graffiti was obtained.

The number of anti-graffiti layers to be applied as well as the drying time between two layers were those recommended by the manufacturer in the technical data sheet of each commercial product. Table 3 shows the number of layers applied in each case:

Tabla 3 / Table 3

Número de capas aplicadas por tipo de anti-grafiti.

Number of coatings applied with each anti-graffiti.

\begin{tabular}{|c|c|c|c|}
\hline Anti-grafiti & Identificación / Identification & Familia / Family & $\mathbf{N}^{\mathbf{0}}$ capas / $\mathbf{N}^{\mathbf{0}}$ coatings \\
\hline Anti-grafiti 1 & $\mathrm{AG}-1$ & $\begin{array}{c}\text { Copolímero de acrilato / } \\
\text { Acrylate copolymer }\end{array}$ & $\begin{array}{c}3 \text { en todos los casos menos en TRAV y BLUE } \\
\text { donde se aplicaron 2 / } 3 \text { in all cases except in } \\
\text { TRAV and BLUE where 2 were applied }\end{array}$ \\
\hline Anti-grafiti 2 & $\mathrm{AG}-2$ & $\begin{array}{c}\text { Polímeros parafínicos / } \\
\text { Paraffin polymers }\end{array}$ & 2 \\
\hline Anti-grafiti 3 & $\mathrm{AG}-3$ & Poliuretano / Polyurethane & 2 \\
\hline Anti-grafiti 4 & $\mathrm{AG}-4$ & Etil metacrilato / Ethyl methacrilate & 2 \\
\hline
\end{tabular}

\subsection{Ensayos efectuados}

Sobre los 8 sustratos tratados con los 4 tipos de anti-grafiti comerciales, así como sobre las muestras patrón, se efectuaron los ensayos que se indican en la Tabla 4, con el fin de estudiar las diferencias entre las muestras con respecto a sus propiedades hídricas y su aspecto. Los ensayos se llevaron a cabo mediante los siguientes procedimientos normalizados:

\subsection{Test procedures}

Table 4 shows the tests performed on the 8 substrates treated with the 4 commercial anti-graffiti and on the blank samples (untreated specimens), with the aim of studying the differences between the samples with regard to its hydric properties and aspect. The tests were carried out following standardized procedures:

Tabla 4 / Table 4

Ensayos realizados y procedimientos utilizados. Tests performed and procedures used.

\begin{tabular}{|c|c|c|}
\hline Propiedad / Property & Ensayo / Test & $\begin{array}{l}\text { Procedimiento normalizado / } \\
\text { Standardized procedure }\end{array}$ \\
\hline \multirow{4}{*}{$\begin{array}{l}\text { Propiedades hídricas / } \\
\text { Hydric properties }\end{array}$} & $\begin{array}{l}\text { Absorción de agua a presión atmosférica / } \\
\text { Water absorption at atmospheric pressure }\end{array}$ & EN 13755 \\
\hline & $\begin{array}{l}\text { Absorción de agua por capilaridad / } \\
\text { Capillary water absorption }\end{array}$ & EN 1925 \\
\hline & $\begin{array}{c}\text { Permeabilidad al vapor de agua / } \\
\text { Water vapour permeability }\end{array}$ & EN ISO 7783-2 \\
\hline & $\begin{array}{c}\text { Comportamiento frente al secado / } \\
\text { Drying behaviour }\end{array}$ & RILEM 25 PEM, II.5 \\
\hline \multirow{2}{*}{$\begin{array}{l}\text { Propiedades físicas: aspecto / } \\
\text { Physical properties: aspect }\end{array}$} & Color / Colour & ISO $7724-2,3$ \\
\hline & Brillo / Gloss & EN ISO 2813 \\
\hline
\end{tabular}

Las medidas de color se realizaron con un colorímetro triestímulo MINOLTA CHROMA METER CR-331C y las medidas de brillo con un brillómetro especular Gardener Glossgards 7500, ángulo 850, tal y como recomienda la norma para recubrimientos de bajo brillo.
The colour measurements were performed using a MINOLTA CHROMA METER CR-331C tristimulus colorimeter and the gloss measurements were performed with a Gardener Glossgards 7500 specular glossmeter. 


\section{RESULTADOS Y DISCUSIÓN}

A partir de las diferencias de peso de las muestras antes y después de la aplicación del anti-grafiti se obtuvo información sobre la cantidad de anti-grafiti de cada tipo que cada sustrato es capaz de absorber. En la Tabla 5 se presentan los resultados de las cantidades de cada anti-grafiti absorbidas por sustrato estudiado:

\section{RESULTS AND DISCUSSION}

The differences in weight of the samples after antigraffiti application provided information on the amount of anti-graffiti that each type of substrate can absorb. Table 5 shows the results of the amounts of anti-graffiti absorbed by each studied substrate:

Tabla 5 / Table 5

Cantidad de anti-grafiti absorbida ( $\mathrm{g}$ ) por cada sustrato estudiado. Absorbed anti-graffiti quantity $(g)$ per studied substrate.

\begin{tabular}{|c|c|c|c|c|c|c|c|c|}
\hline \multirow[b]{2}{*}{ Sustrato / Substrate } & \multicolumn{2}{|c|}{ AG-1 } & \multicolumn{2}{|c|}{ AG-2 } & \multicolumn{2}{|c|}{ AG-3 } & \multicolumn{2}{|c|}{ AG-4 } \\
\hline & $\begin{array}{l}\text { Valor medio I } \\
\text { Average value }\end{array}$ & $\sigma$ & $\begin{array}{l}\text { Valor medio I } \\
\text { Average value }\end{array}$ & $\sigma$ & $\begin{array}{l}\text { Valor medio / } \\
\text { Average value }\end{array}$ & $\sigma$ & $\begin{array}{l}\text { Valor medio / } \\
\text { Average value }\end{array}$ & $\sigma$ \\
\hline VILLA & 626.8 & 51.0 & 586.9 & 84.5 & 427.3 & 25.5 & 633.9 & 58.7 \\
\hline GALD & 246.9 & 38.9 & 194.7 & 33.3 & 322.9 & 14.7 & 356.1 & 105.3 \\
\hline STRT & 226.8 & 45.3 & 143.6 & 11.7 & 331.1 & 25.8 & 289.1 & 46.7 \\
\hline BAUM & 223.0 & 33.4 & 105.8 & 23.4 & 272.1 & 45.3 & 164.4 & 38.7 \\
\hline BLUE & 52.9 & 23.4 & 60.5 & 18.5 & 173.8 & 31.0 & 204.1 & 32.1 \\
\hline BALE & 158.7 & 37.9 & 124.7 & 23.8 & 223.0 & 33.4 & 368.5 & 79.4 \\
\hline TRAV & 49.1 & 17.1 & 196.5 & 34.1 & 234.3 & 60.3 & 147.4 & 54.0 \\
\hline LADR & 540.4 & 59.8 & 619.8 & 151.5 & 506.4 & 88.0 & 515.9 & 77.2 \\
\hline
\end{tabular}

De acuerdo a estos resultados, en general se puede decir que las cantidades de anti-grafiti absorbidas fueron menores en el caso de los sustratos de menor porosidad (Caliza azul belga: $52,9-204,1 \mathrm{~g} / \mathrm{m}^{2}$ ), mientras que los sustratos con una porosidad alta y mayor tamaño de poro fueron los que más cantidad de producto absorbieron (ladrillo: 506,4 - 619,8 g/m²; arenisca silícea de Villamayor: 427,3-633,9 g/m²). Además, en base a los valores obtenidos, puede decirse que el tamaño de poro es el factor que determina, más que la porosidad, la absorción de anti-grafiti. Cabe mencionar, asimismo, que en general las cantidades de los anti-grafiti 3 y 4 absorbidas fueron mayores que los de los anti-grafiti 1 y 2 , observándose que el anti-grafiti 2 parece ser el producto que menos se absorbe.

Los resultados obtenidos en los diferentes ensayos de medida de las propiedades hídricas y físicas permitieron comparar el comportamiento de los diferentes anti-grafiti entre sí, así como su comportamiento con respecto al sustrato no tratado.

La Tabla 6 muestra los resultados obtenidos en los ensayos de absorción de agua a presión atmosférica, succión capilar, permeabilidad al vapor de agua y comportamiento frente al secado.

La idoneidad de los anti-grafiti y otros productos de recubrimiento para su aplicación en sustratos porosos, se determina en primer lugar por el comportamiento hídrico de dichos materiales cuando se encuentran recubiertos por
According to these results and as expected, the absorbed anti-graffiti amounts were lower in the case of the lower porosity substrates (Belgian blue limestone: 52.9 - 204.1 $\left.\mathrm{g} / \mathrm{m}^{2}\right)$, whereas the substrates presenting high porosity and bigger pore size were those absorbing more product (Brick: 506.4 - $619.8 \mathrm{~g} / \mathrm{m}^{2}$; Villamayor siliceous sandstone: $427.3-633.9 \mathrm{~g} / \mathrm{m}^{2}$ ). Based on the results obtained, it can be said that the pore size is the factor determining the anti-graffiti absorption, more than porosity. It must also be mentioned that the absorbed amounts in the case of anti-graffiti 3 and 4 were higher than those of anti-graffiti 1 and 2, being overall antigraffiti 2 the least absorbed product.

The results from the different tests performed to measure the hydric and physical properties permitted to compare the behaviour of the different anti-graffiti among themselves, as well as their behaviour with respect to the untreated substrate.

Table 6 presents the results of the tests performed to measure the water absorption at atmospheric pressure, capillary suction, water vapour permeability and drying behaviour.

The suitability of anti-graffiti and other surface treatments for their application in porous substrates is initially determined by the hydric behaviour of these porous materials when they are treated with the 
las capas protectoras mencionadas $(10,11)$. Puesto que todos los materiales porosos que constituyen los elementos exteriores de los edificios presentan una cierta cantidad de agua que circula a través de su red porosa, y que se intercambia, en cierta medida, con el ambiente exterior, dichos recubrimientos deben permitir que la circulación e intercambio de agua sea tal que no cause el deterioro de dicho material (7), pero que a su vez sea capaz de protegerlo de las agresiones externas (12). De entre las propiedades hídricas más determinantes, caben destacar la permeabilidad al vapor de agua y el comportamiento frente al secado, tal y como ya explicaron otros autores como Ashurst et al. o Lubelli et al. $(12,13)$. Aquellos recubrimientos que alteran significativamente los valores de dichas propiedades pueden causar deterioros importantes en el sustrato, por lo que las reducciones que confieren en el sustrato a tratar deben ser estudiadas con detenimiento antes de realizar la aplicación final del producto (14). protective coatings mentioned $(10,11)$. As all porous materials that form the outdoor elements of buildings present a certain water amount circulating through their pore system, and considering that this water is exchanged, to a certain extent, with the outdoor environment, such treatments must permit free water circulation and exchange so as not to cause any deterioration to these materials (7) but enabling protection from external aggressions (12). Among the most determining hydric properties water vapour permeability and drying behaviour must be highlighted, as Ashurst et al. or Lubelli et al. explained $(12,13)$. Treatments significantly altering the values of such properties can cause important damages, thus the reductions they confer to the substrate to be treated should be carefully studied before performing the final product application (14).

Tabla 6 / Table 6

Resultados de las medidas de las propiedades hídricas.

Results of the measurement of the hydric properties.

\begin{tabular}{|c|c|c|c|c|c|c|c|}
\hline $\begin{array}{l}\text { Propiedad / } \\
\text { Property }\end{array}$ & $\begin{array}{l}\text { Parámetro I } \\
\text { Parameter }\end{array}$ & $\begin{array}{l}\text { Sustrato / } \\
\text { Substrate }\end{array}$ & $\begin{array}{c}\text { Patrón I } \\
\text { Blank }\end{array}$ & AG-1 & AG-2 & AG-3 & AG-4 \\
\hline \multirow{8}{*}{$\begin{array}{l}\text { Absorción de agua a } \\
\text { presión atmosférica / } \\
\text { Water absorption at } \\
\text { atmospheric pressure }\end{array}$} & \multirow{8}{*}{$\begin{array}{c}\text { Absorción de agua a } \\
\text { presión atmosférica / } \\
\text { Water absorption at } \\
\text { atmospheric pressure } \\
(\%)\end{array}$} & VILLA & 13.8 & 13.5 & 12.9 & 3.0 & 12.2 \\
\hline & & GALD & 4.7 & 5.2 & 5.1 & 0.3 & 5,1 \\
\hline & & BALE & 2.1 & 2.5 & 2.6 & 1.7 & 2.0 \\
\hline & & BLUE & 0.1 & 0.02 & 0.1 & 0.1 & 0.1 \\
\hline & & STRT & 4.7 & 4,0 & 4.0 & 0.1 & 4.0 \\
\hline & & TRAV & 1.6 & 1.1 & 1.4 & 0.8 & 0.4 \\
\hline & & BAUM & 5.9 & 5.9 & 5.6 & 5.8 & 5.3 \\
\hline & & LADR & 13.2 & 12.1 & 12.8 & 13.2 & 13.1 \\
\hline \multirow{8}{*}{$\begin{array}{l}\text { Succión capilar / } \\
\text { Capillary suction }\end{array}$} & \multirow{8}{*}{$\begin{array}{c}\text { Coeficiente de absorción } \\
\text { de agua por capilaridad / } \\
\text { Capillary water } \\
\text { absorption coefficient } \\
\left(\mathrm{g} / \mathrm{m}^{2} \mathrm{~s}^{1 / 2}\right)\end{array}$} & VILLA & 68.2 & 25.8 & 31.6 & 2.10 & 7.58 \\
\hline & & GALD & 27.0 & 19.5 & 15.9 & 1.30 & 2.41 \\
\hline & & STRT & 12.8 & 10.8 & 11.6 & 3.13 & 12.4 \\
\hline & & BAUM & 21.0 & 21.6 & 14.7 & 0.843 & 1.08 \\
\hline & & BLUE & 0.143 & 0.0966 & 0.0842 & 0.189 & 0.212 \\
\hline & & BALE & 3.63 & 1.22 & 1.30 & 0.153 & 0.411 \\
\hline & & TRAV & 2.48 & 0.886 & 2.76 & 1.61 & 0.222 \\
\hline & & LADR & 260 & 102 & 55.1 & 26.3 & 16.6 \\
\hline \multirow{8}{*}{$\begin{array}{c}\text { Permeabilidad al vapor } \\
\text { de agua / } \\
\text { Water vapour } \\
\text { permeability }\end{array}$} & \multirow{8}{*}{$\begin{array}{c}\text { Velocidad de } \\
\text { transmisión de } \\
\text { agua-vapor / } \\
\text { Water vapour } \\
\text { transmisión rate } \\
\left(\mathrm{g} / \mathrm{m}^{2} \mathrm{~d}\right)\end{array}$} & VILLA & 23.01 & 12.97 & 14.02 & 8.68 & 14.22 \\
\hline & & GALD & 14.07 & 10.16 & 11.85 & 9.98 & 10.55 \\
\hline & & STRT & 71.02 & 50.24 & 52.85 & 8.93 & 43.87 \\
\hline & & BAUM & 101.22 & 62.73 & 60.52 & 11.94 & 44.38 \\
\hline & & BLUE & 0.38 & 0.15 & 0.16 & 0.11 & 0.14 \\
\hline & & BALE & 1.93 & 1.62 & 1.97 & 0.43 & 0.77 \\
\hline & & TRAV & 72.16 & 86.14 & 71.99 & 28.26 & 31.51 \\
\hline & & LADR & 108.27 & 90.85 & 56.68 & 24.91 & 64.74 \\
\hline \multirow{8}{*}{$\begin{array}{l}\text { Comportamiento frente } \\
\text { al secado / } \\
\text { Drying behaviour }\end{array}$} & \multirow{8}{*}{$\begin{array}{c}\text { Evaporación a } 72 \mathrm{~h} / \\
\text { Evaporation at } 72 \mathrm{~h} \\
\left(\mathrm{~g} / \mathrm{m}^{2}\right)\end{array}$} & VILLA & 4712 & 3381 & 3682 & 392 & 3652 \\
\hline & & GALD & 3428 & 3859 & 2477 & 81 & 1726 \\
\hline & & STRT & 2121 & 1979 & 2179 & 152 & 951 \\
\hline & & BAUM & 4019 & 3700 & 570 & 83 & 726 \\
\hline & & BLUE & 30 & 24 & 17 & 14 & 16 \\
\hline & & BALE & 1356 & 748 & 574 & 55 & 327 \\
\hline & & TRAV & 558 & 302 & 476 & 253 & 172 \\
\hline & & LADR & 5476 & 3970 & 4273 & 308 & 1392 \\
\hline
\end{tabular}


En la Tabla 6 se presentan los resultados obtenidos en las diferentes propiedades hídricas que se midieron.

Los resultados obtenidos demuestran que todos los productos anti-grafiti aplicados reducen las propiedades hídricas de los sustratos estudiados, es decir, el intercambio libre de agua tanto en fase vapor como en fase líquida se ve impedida. En cambio, estas reducciones varían con el tipo de anti-grafiti aplicado. Debe mencionarse que cuando los valores de las muestras tratadas superan los de las muestras no tratadas, se considera que la irregularidad se debe a las heterogeneidades que puede presentar la muestra ensayada.

Los resultados de succión capilar indican que la aplicación de anti-grafiti reduce la capacidad de succión natural del sustrato, dependiendo esta reducción, por un lado, de la composición química del recubrimiento, y por otro, de la capacidad de succión en sí del sustrato, la cual a su vez depende de la porosidad y del tamaño de poro, y de la interconexión de poros. Así, los sustratos que presentan una capacidad de succión más rápida (caso del ladrillo, coeficiente de absorción de agua por capilaridad: $260 \mathrm{~g} / \mathrm{m}^{2} \mathrm{~s}^{1 / 2}$; porosidad: $19,5 \%$; tamaño de poro; $0,3 \mu \mathrm{m})$, presentan también mayores reducciones en la succión tras la aplicación del producto anti-grafiti. En cambio, a los sustratos que succionan más lentamente (caso de la arenisca calcárea de Strtenica, por ejemplo), la aplicación de anti-grafiti les afecta menos, pues se observa que las reducciones son menores. Si los antigrafiti son comparados entre sí, se puede concluir que el anti-grafiti que mayor reducción de la succión capilar produce en los sustratos es el anti-grafiti 3 (poliuretano), clasificado como permanente. Tal y como ya comentaron Lubelli et al. (13), este tipo de productos producen una capa impermeable que impiden la entrada y salida libre de agua, dando lugar a reducciones en la mayoría de los casos mayores del $90 \%$. Es por esto que este tipo de productos no son recomendados en la protección de sustratos porosos, y son normalmente aplicados en materiales metálicos, vidrio, etc. Por el contrario, los anti-grafiti 1 (copolímero de acrilato) y 2 (polímeros parafínicos) estudiados, clasificados como de sacrificio de acuerdo a su composición y ficha técnica del producto, presentan las reducciones más bajas. El anti-grafiti 4 (metacrilato de etilo) presenta un comportamiento similar al anti-grafiti 3, pero con reducciones menores.

Los valores de velocidad de transmisión de agua-vapor también presentan reducciones significativas tras la aplicación de producto anti-grafiti. Esta propiedad es aquella que más importancia recibe por parte de los conservadores, pues la idoneidad de los productos de conservación como consolidantes, hidrofugantes o anti-grafiti depende en gran medida de su permeabilidad al vapor de agua. Los resultados demuestran que las reducciones más significativas son
In Table 6 the results obtained in the different measured hydric properties are presented.

The results obtained show that all applied anti-graffiti products reduce the hydric properties of the studied substrates i.e. the free water exchange both in the vapour phase and in the liquid phase is hindered. However, these reductions vary depending on the type of anti-graffiti applied. It must be mentioned that the treated samples presenting higher values in comparison to the untreated samples are considered to be due to the heterogeneities that the sample might present.

The results of the capillary suction indicate that the antigraffiti application reduces the natural ability of the substrate to suction. This reduction depends on the one hand on the chemical composition of the coating, and on the other hand on the ability of the substrate to suction, which depends at the same time on the porosity and pore size, and the pore interconnection. In this sense, the substrates presenting greater suction ability (like in the case of the brick, capillary water absorption coefficient: $260 \mathrm{~g} / \mathrm{m}^{2} \mathrm{~s}^{1 / 2}$; porosity: 19.5\%; pore size; $0.3 \mu \mathrm{m})$, also present higher suction reductions after the application of the anti-graffiti product. However, the substrates suctioning more slowly (like in the case of the Strtenica calcareous substrate, for instance) are less affected by the anti-graffiti application as it is observed that the reductions are lower. When the anti-graffiti are compared, it can be concluded that the anti-graffiti producing higher reductions in the capillary water absorption of the substrates is anti-graffiti 3 (polyurethane), classified as permanent. According to Lubelli et al. (13) this kind of products form an impermeable coating which impedes the free water uptake and outtake, leading to reductions higher than $90 \%$ in most of the cases. For this reason, this type of products are not recommended for the protection of porous substrates and are normally applied in metallic materials, glass, etc. On the contrary, the studied antigraffiti 1 (acrylate copolymer) and 2 (paraffinic polymers), which are classified as sacrificial according to their composition and product technical data sheets, present the lower reductions. The anti-graffiti 4 (ethyl methacrylate) shows a similar behaviour to that of antigraffiti 3, but presents lower reductions.

The water vapour transmission rate values also present significant reductions after the anti-graffiti product application. This property is considered to be the most important by conservators as the suitability of the conservation products such as consolidants, water repellents or anti-graffiti depends to a large extent on its water vapour permeability. The results confirm that the most significant reductions are conferred by anti-graffiti 
conferidas por el anti-grafiti 3 (poliuretano), en consonancia con los resultados de succión capilar discutidos en el párrafo anterior. Asimismo, los anti-grafiti de sacrificio 1 y 2 son los que menores reducciones en la velocidad de transmisión de agua-vapor presentan, siendo el comportamiento del anti-grafiti 2 (polímeros parafínicos) ligeramente mejor que el del anti-grafiti 1 (copolímero de acrilato). La habilidad de los anti-grafiti en base parafínica y acrílica de permitir el paso del agua en fase vapor fue ya estudiada por Ashurst et al. (7). Así, se observa en los resultados obtenidos que las reducciones son menores en los sustratos de porosidad alta, pues en estos sustratos la velocidad de transmisión de agua-vapor es de por sí más alta.

En lo que al comportamiento frente al secado se refiere, se escogieron los valores de evaporación de agua de todos los tipos de sustratos tras $72 \mathrm{~h}$ de ensayo y se compararon entre sí. Esta propiedad también debe tenerse muy en cuenta a la hora de evaluar la idoneidad del producto de conservación a aplicar sobre el sustrato poroso protegido, pues la efectividad del mismo dependerá en gran medida en lo seca o húmeda que esté la superficie a tratar (15). El secado de un material poroso es un proceso complejo que comporta fases diferentes, según describieron Wendler y Charola (16). Los resultados obtenidos en este estudio vuelven a confirmar que los anti-grafiti que en menor medida reducen la velocidad de secado del sustrato son los dos productos de sacrificio estudiados (anti-grafiti 1 y 2), mientras que el poliuretano (anti-grafiti 3) es el producto que más reduce el secado natural del sustrato (de más del $90 \%$ en la mayoría de los casos).

Finalmente, puede decirse que los resultados de absorción de agua a presión atmosférica no permiten establecer diferencias significativas entre los diferentes anti-grafiti, e incluso en comparación al sustrato no tratado, lo cual puede deberse al tipo de ensayo elegido, pues solamente se aplicó anti-grafiti en una de las caras de las muestras.

A pesar de que las características hídricas resultan totalmente condicionantes a la hora de establecer la idoneidad de los tratamientos anti-grafiti sobre superficies porosas, también el aspecto que estos productos confieren a la superficie tratada, resulta de vital importancia, fundamentalmente, por el valor histórico que tienen las superficies que se tratan.

En la Tabla 7 se presentan los valores de color, medidos de acuerdo a la norma descrita en la sección 2, para cada uno de los sustratos porosos y productos anti-grafiti analizados.

Se midieron mediante colorímetro triestímulo la luminosidad $\left(\mathrm{L}^{*}\right)$ y las coordenadas de color ( $\mathrm{a}^{*}$ y $\mathrm{b}^{*}$ ) de las muestras antes de la aplicación de anti-grafiti y después de la aplicación de anti-grafiti. Para evaluar las
3 (polyurethane), in tune with the results of capillary suction discussed in the previous paragraph. In this line, the sacrificial anti-graffiti 1 and 2 are the ones presenting the lowest reductions in the water vapour transmission rate, being the behaviour of anti-graffiti 2 (paraffin polymers) slightly better than that of anti-graffiti 1 (acrylate copolymer). The ability of the paraffin- and acrylic-based anti-graffiti to permit the water vapour transmission was studied by Ashurst et al. (7). From the results obtained it is observed that the reductions are lower in the high porosity substrates, because in these substrates the water vapour transmission rate is originally higher.

With regard to the drying behaviour, the water evaporation values of all types of substrates after $72 \mathrm{~h}$ of test were considered and these values compared. This property has to be taken into account too when evaluating the suitability of a conservation product for its application on a protected porous substrate, as its effectiveness will depend to a large extent on how dry or wet the surface to be treated is (15). The drying of a material is complex process which involves different phases, as described by Wendler and Charola (16). The results obtained in this study confirm once more that the anti-graffiti reducing to a lesser extent the drying rate of the substrate are the two sacrificial products studied (anti-graffiti 1 and 2), whereas the polyurethane (antigraffiti 3) is the product most reducing the natural drying of the substrates (higher than $90 \%$ in most of the cases).

Finally, it can be said that the results of water absorption at atmospheric pressure do not permit to establish significant differences among the different anti-graffiti, nor in comparison with the untreated substrate. This can be due to the type of test selected because the anti-graffiti was applied only in one of the sides of the samples.

Although the hydric properties are conditioning to a large extent the suitability of the anti-graffiti treatments for their application on porous substrates, the aspect that these products confer to the treated surface is of vital importance mainly due to the historical value that these surfaces present.

In the Table 7 the values of colour are presented. These were measured according to the standard described in section 2, for each of the porous substrates and antigraffiti products analyzed.

By means of a tristimulus colorimeter the lightness $\left(L^{*}\right)$ and the colour coordinates ( $a^{*}$ and $b^{*}$ ) were measured before and after the application of anti-graffiti. In order to evaluate the differences in colour among the samples, 
diferencias de color entre muestras, se calculó $\Delta \mathrm{E}^{*} \mathrm{a}, \mathrm{b}$ (diferencia de color total) de acuerdo a la siguiente ecuación [1] (norma ISO 7724-3):
$\Delta E^{*}$ a, (total colour difference) according to the following equation [1] (standard ISO 7724-3):

$$
\Delta \mathrm{E}^{*}{ }_{\mathrm{a}, \mathrm{b}}=\left(\left(\Delta \mathrm{a}^{*}\right)^{2}+\left(\Delta \mathrm{b}^{*}\right)^{2}+\left(\Delta \mathrm{L}^{*}\right)^{2}\right)^{1 / 2}
$$

Siendo:

Being:

$$
\Delta a^{*}=a^{*}{ }_{2}-a^{*}{ }_{1} ; \Delta b^{*}=b^{*}{ }_{2}-b^{*}{ }_{1} ; \Delta L^{*}=L^{*}{ }_{2}-L^{*}{ }_{1}
$$

Donde:

$a *_{1}, b^{*}{ }_{1}$ y $L^{*}{ }_{1}$ son los valores de las coordenadas de color ( $a^{*}$ y $\left.b^{*}\right)$ y de luminosidad $\left(L^{*}\right)$ antes de la aplicación de anti-grafiti;

\section{Where:}

$a *_{1}, b^{*}{ }_{1}$ y $L^{*}{ }_{1}$ are the values of the colour coordinates ( $a^{*}$ and $b^{*}$ ) and of the lightness $\left(L^{*}\right)$ before the application of anti-graffiti;

Tabla 7 / Table 7

Resultados de las medidas de color.

\begin{tabular}{|c|c|c|c|c|c|c|}
\hline $\begin{array}{c}\text { Propiedad / } \\
\text { Property }\end{array}$ & $\begin{array}{l}\text { Sustrato / } \\
\text { Substrate }\end{array}$ & Patrón / Blank & AG-1 & AG-2 & AG-3 & AG-4 \\
\hline \multirow{8}{*}{$\begin{array}{l}\text { Color / Colour } \\
\qquad \begin{array}{c}\left(\Delta \mathrm{L}^{*}\right) \\
\left(\Delta \mathrm{a}^{*}\right) \\
\left(\Delta \mathrm{b}^{*}\right) \\
\left(\Delta \mathrm{E}^{*} \mathrm{a}, \mathrm{b}\right)\end{array}\end{array}$} & VILLA & $\begin{array}{c}L^{*}: 70.90 \\
a^{*}: 1.90 \\
b^{*}: 14.43\end{array}$ & $\begin{array}{c}\Delta \mathrm{L}^{*}:-0.83 \\
\Delta \mathrm{a}^{*}: 0.07 \\
\Delta \mathrm{b}^{*}: 2.74 \\
\Delta \mathrm{E}^{*} \mathrm{a}, \mathrm{b}: 2.85 \\
\text { Clas: } \mathrm{A}\end{array}$ & $\begin{array}{c}\Delta \mathrm{L}^{*}: 1.35 \\
\Delta \mathrm{a}^{*}:-0.21 \\
\Delta \mathrm{b}^{*}: 1.38 \\
\Delta \mathrm{E}^{*} \mathrm{a}, \mathrm{b}: 1.92 \\
\text { Clas.: A }\end{array}$ & $\begin{array}{c}\Delta \mathrm{L}^{*}: 11.66 \\
\Delta \mathrm{a}^{*}: 2.87 \\
\Delta \mathrm{b}^{*}: 7.15 \\
\Delta \mathrm{E}^{*} \mathrm{a}, \mathrm{b}: 14.04 \\
\text { Clas.: B }\end{array}$ & $\begin{array}{c}\Delta \mathrm{L}^{*}:-9.26 \\
\Delta \mathrm{a}^{*}: 2.31 \\
\Delta \mathrm{b}^{*}: 7.99 \\
\Delta \mathrm{E}^{*} \text { a,b: } 12.45 \\
\text { Clas.: B }\end{array}$ \\
\hline & GALD & $\begin{array}{c}L^{*}: 73.63 \\
a^{*}: 1.43 \\
b^{*}: 12.78\end{array}$ & $\begin{array}{c}\Delta \mathrm{L}^{*}:-4.09 \\
\Delta \mathrm{a}^{*}: 1.00 \\
\Delta \mathrm{b}^{*}: 5.04 \\
\Delta \mathrm{E}^{*} \text { a,b: } 6.49 \\
\text { Clas.: } \mathrm{B}\end{array}$ & $\begin{array}{c}\Delta \mathrm{L}^{*}:-3.69 \\
\Delta \mathrm{a}^{*}: 0.70 \\
\Delta \mathrm{b}^{*}: 3.54 \\
\Delta \mathrm{E}^{*} \text { a,b: } 5.16 \\
\text { Clas.: } \mathrm{B}\end{array}$ & $\begin{array}{c}\Delta \mathrm{L}^{*}:-14.76 \\
\Delta \mathrm{a} *: 2.92 \\
\Delta \mathrm{b}^{*}: 5.04 \\
\Delta \mathrm{E}^{*} \mathrm{a}, \mathrm{b}: 15.90 \\
\text { Clas.: B }\end{array}$ & $\begin{array}{c}\Delta \mathrm{L}^{*}:-10.85 \\
\Delta \mathrm{a}^{*}: 2.61 \\
\Delta \mathrm{b}^{*}: 6.52 \\
\Delta \mathrm{E}^{*} \text { a,b: } 12.94 \\
\text { Clas.: B }\end{array}$ \\
\hline & STRT & $\begin{array}{c}L^{*}: 70.52 \\
a^{*}: 0.36 \\
b^{*}: 11.87\end{array}$ & $\begin{array}{c}\Delta \mathrm{L}^{*}:-4.71 \\
\Delta \mathrm{a} *: 0.83 \\
\Delta \mathrm{b}^{*}: 3.63 \\
\Delta \mathrm{E}^{*} \mathrm{a}, \mathrm{b}: 6.01 \\
\text { Clas.: B }\end{array}$ & $\begin{array}{c}\Delta \mathrm{L}^{*}:-1.75 \\
\Delta \mathrm{a}^{*}: 0.48 \\
\Delta \mathrm{b}^{*}: 1.61 \\
\Delta \mathrm{E}^{*} \text { a,b: } 2.43 \\
\text { Clas.: A }\end{array}$ & $\begin{array}{c}\Delta \mathrm{L}^{*}:-20.75 \\
\Delta \mathrm{a}^{*}: 2.34 \\
\Delta \mathrm{b}^{*}:-1.04 \\
\Delta \mathrm{E}^{*} \text { a, b: } 20.92 \\
\text { Clas.: B }\end{array}$ & $\begin{array}{c}\Delta \mathrm{L}^{*}:-12.24 \\
\Delta \mathrm{a}^{*}: 1.83 \\
\Delta \mathrm{b}^{*}: 5.41 \\
\Delta \mathrm{E}^{*} \text { a,b: } 13.54 \\
\text { Clas.: B }\end{array}$ \\
\hline & BAUM & $\begin{array}{c}L^{*}: 72.97 \\
a^{*}: 0.29 \\
b^{*}: 10.60\end{array}$ & $\begin{array}{c}\Delta \mathrm{L}^{*}:-5.12 \\
\Delta \mathrm{a} *: 0.82 \\
\Delta \mathrm{b}^{*}: 5.55 \\
\Delta \mathrm{E}^{*} \mathrm{a}, \mathrm{b}: 7.61 \\
\text { Clas.: B }\end{array}$ & $\begin{array}{c}\Delta \mathrm{L}^{*}:-0.80 \\
\Delta \mathrm{a}^{*}: 0.19 \\
\Delta \mathrm{b}^{*}: 1.55 \\
\Delta \mathrm{E}^{*} \text { a,b: } 1.78 \\
\text { Clas.: A }\end{array}$ & $\begin{array}{c}\Delta \mathrm{L}^{*}:-22.03 \\
\Delta \mathrm{a}^{*}: 2.68 \\
\Delta \mathrm{b}^{*}: 5.20 \\
\Delta \mathrm{E}^{*} \mathrm{a}, \mathrm{b}: 22.80 \\
\text { Clas.: B }\end{array}$ & $\begin{array}{c}\Delta \mathrm{L}^{*}:-10.55 \\
\Delta \mathrm{a}^{*}: 1.86 \\
\Delta \mathrm{b}^{*}: 6.21 \\
\Delta \mathrm{E}^{*} \text { a, b: } 12.42 \\
\text { Clas.: } \mathrm{B}\end{array}$ \\
\hline & BLUE & $\begin{array}{l}L^{*}: 55.44 \\
a^{*}:-0.69 \\
b^{*}:-1.17\end{array}$ & $\begin{array}{c}\Delta \mathrm{L}^{*}:-16.89 \\
\Delta \mathrm{a}^{*}: 0.83 \\
\Delta \mathrm{b}^{*}: 1.83 \\
\Delta \mathrm{E}^{*} \text { a,b: } 17.01 \\
\text { Clas.: B }\end{array}$ & $\begin{array}{c}\Delta \mathrm{L}^{*}:-8.88 \\
\Delta \mathrm{a}^{*}: 0.40 \\
\Delta \mathrm{b}^{*}: 0.97 \\
\Delta \mathrm{E}^{*} \mathrm{a}, \mathrm{b}: 8.94 \\
\text { Clas.: } \mathrm{B}\end{array}$ & $\begin{array}{c}\Delta \mathrm{L}^{*}:-14.30 \\
\Delta \mathrm{a}^{*}: 0.67 \\
\Delta \mathrm{b}^{*}: 1.66 \\
\Delta \mathrm{E}^{*} \mathrm{a}, \mathrm{b}: 14.42 \\
\text { Clas.: B }\end{array}$ & $\begin{array}{c}\Delta \mathrm{L}^{*}:-18.11 \\
\Delta \mathrm{a}^{*}: 0.71 \\
\Delta \mathrm{b}^{*}: 2.52 \\
\Delta \mathrm{E}^{*} \text { a,b: } 18.30 \\
\text { Clas.: B }\end{array}$ \\
\hline & BALE & $\begin{array}{l}L^{*}: 70.87 \\
a^{*}:-0.27 \\
b^{*}: 7.52\end{array}$ & $\begin{array}{c}\Delta \mathrm{L}^{*}:-5.79 \\
\Delta \mathrm{a}^{*}: 0.65 \\
\Delta \mathrm{b}^{*}: 5.04 \\
\Delta \mathrm{E}^{*} \text { a,b: } 7.75 \\
\text { Clas.: B }\end{array}$ & $\begin{array}{c}\Delta \mathrm{L}^{*}:-3.31 \\
\Delta \mathrm{a}^{*}: 0.40 \\
\Delta \mathrm{b}^{*}: 2.12 \\
\Delta \mathrm{E}^{*} \text { a,b: } 3.97 \\
\text { Clas.: A }\end{array}$ & $\begin{array}{c}\Delta \mathrm{L}^{*}:-17.45 \\
\Delta \mathrm{a}^{*}: 1.61 \\
\Delta \mathrm{b}^{*}: 5.64 \\
\Delta \mathrm{E}^{*} \mathrm{a}, \mathrm{b}: 18.45 \\
\text { Clas.: B }\end{array}$ & $\begin{array}{c}\Delta \mathrm{L}^{*}:-12.54 \\
\Delta \mathrm{a}^{*}: 0.65 \\
\Delta \mathrm{b}^{*}: 6.53 \\
\Delta \mathrm{E}^{*} \text { a,b: } 14.17 \\
\text { Clas.: B }\end{array}$ \\
\hline & TRAV & $\begin{array}{c}L^{*}: 81.89 \\
a^{*}: 2.35 \\
b^{*}: 11.87\end{array}$ & $\begin{array}{c}\Delta \mathrm{L}^{*}:-1.13 \\
\Delta \mathrm{a}^{*}: 0.35 \\
\Delta \mathrm{b}^{*}: 3.87 \\
\Delta \mathrm{E}^{*} \text { a,b: } 4.09 \\
\text { Clas.: } \mathrm{B}\end{array}$ & $\begin{array}{c}\Delta \mathrm{L}^{*}:-1.67 \\
\Delta \mathrm{a}^{*}: 0.51 \\
\Delta \mathrm{b}^{*}: 2.81 \\
\Delta \mathrm{E}^{*} \text { a,b: } 3.33 \\
\text { Clas.: A }\end{array}$ & $\begin{array}{c}\Delta \mathrm{L}^{*}:-2.49 \\
\Delta \mathrm{a} *: 0.65 \\
\Delta \mathrm{b}^{*}: 4.75 \\
\Delta \mathrm{E}^{*} \mathrm{a}, \mathrm{b}: 5.41 \\
\text { Clas.: } \mathrm{B}\end{array}$ & $\begin{array}{c}\Delta \mathrm{L}^{*}:-3.35 \\
\Delta \mathrm{a}^{*}: 0.87 \\
\Delta \mathrm{b}^{*}: 5.45 \\
\Delta \mathrm{E}^{*} \mathrm{a}, \mathrm{b}: 6.48 \\
\text { Clas.: B }\end{array}$ \\
\hline & LADR & $\begin{array}{l}L^{*}: 50.87 \\
a^{*}: 22.43 \\
b^{*}: 28.51\end{array}$ & $\begin{array}{c}\Delta \mathrm{L}^{*}:-4.69 \\
\Delta \mathrm{a}^{*}: 2.78 \\
\Delta \mathrm{b}^{*}: 1.48 \\
\Delta \mathrm{E}^{*} \text { a,b: } 5.68 \\
\text { Clas.: B }\end{array}$ & $\begin{array}{c}\Delta \mathrm{L}^{*}:-2.44 \\
\Delta \mathrm{a}^{*}: 1.99 \\
\Delta \mathrm{b}^{*}: 1.90 \\
\Delta \mathrm{E}^{*} \mathrm{a}, \mathrm{b}: 4.56 \\
\text { Clas.: } \mathrm{B}\end{array}$ & $\begin{array}{c}\Delta \mathrm{L}^{*}:-6.36 \\
\Delta \mathrm{a}^{*}:-0.89 \\
\Delta \mathrm{b}^{*}:-8.56 \\
\Delta \mathrm{E}^{*} \text { a,b: } 10.90 \\
\text { Clas.: B }\end{array}$ & $\begin{array}{c}\Delta \mathrm{L}^{*}:-8.46 \\
\Delta \mathrm{a}^{*}: 1.60 \\
\Delta \mathrm{b}^{*}:-4.13 \\
\Delta \mathrm{E}^{*} \text { a,b: } 9.56 \\
\text { Clas.: B }\end{array}$ \\
\hline
\end{tabular}

Results of the measurements of colour.

Clas.: clasificación / classification. 
$a *_{2}, b *_{2}$ y $L^{*}{ }_{2}$ son los valores de las coordenadas de color $\left(a^{*}\right.$ y $\left.b^{*}\right)$ y de luminosidad $\left(L^{*}\right)$ después de la aplicación de anti-grafiti.

De acuerdo a la clasificación de valores de $\Delta \mathrm{E}^{*} \mathrm{a}, \mathrm{b}$ propuesta por Quenard (17), se evaluaron los resultados de dicho parámetro estableciendo el límite de 4 unidades de color para aquellos cambios que no son perceptibles para el ojo humano (clasificación A). En cambio, cuando $\Delta \mathrm{E}^{*}$ a,b fue mayor de 4 unidades, se consideró que el cambio de color entre la muestra no tratada y la tratada era perceptible (clasificación B).

Los resultados indican que los sustratos más oscuros son el ladrillo y la caliza azul belga (L: 50,87 y 55,44 respectivamente), mientras que el más claro o luminoso es el travertino romano (L: 81,89). Así, en la mayoría de los casos, la aplicación de anti-grafiti oscurece la muestra $\left(\Delta L^{*}<0\right)$, siendo en general los anti-grafiti 3 (poliuretano) y 4 (metacrilato de etilo) los productos que más oscurecen los sustratos. Esto puede deberse a la mayor cantidad de producto absorbido por los sustratos, tal y como ya se ha comentado anteriormente, en el caso de los anti-grafiti 3 y 4 . Las diferencias de color total $\left(\Delta \mathrm{E}^{*} \mathrm{a}, \mathrm{b}\right)$ son perceptibles en todos los sustratos tras la aplicación de estos 2 productos, siendo los sustratos más afectados las areniscas calcáreas en el caso del anti-grafiti 3 y la caliza azul belga en el caso del anti-grafiti 4. En cambio, el anti-grafiti con base parafínica (anti-grafiti 2) presenta una diferencia de color total mayor de 4 unidades únicamente en la caliza azul belga, la arenisca silícea de Galdakao y el ladrillo, siendo la luminosidad el parámetro que más varía en los tres casos. Sin embargo cabe destacar que, excepto en el caso de la caliza azul, las diferencias de color total no son significativas. Finalmente, el anti-grafiti 1 (copolímero de acrilato) solamente produce cambios no perceptibles en un único sustrato de los 8 estudiados, como es la arenisca silícea de Villamayor, pero en general, los valores de $\Delta \mathrm{E}^{*}$ a,b son menores en comparación a los de los anti-grafiti 3 y 4.

Con respecto a los cambios en el brillo conferidos por la aplicación de anti-grafiti, la Tabla 8 muestra las diferencias de brillo entre las medidas realizadas antes y después de la aplicación de cada anti-grafiti:

Para la evaluación del brillo, se consideró que una diferencia menor de 3 unidades con respecto a la muestra no tratada no es perceptible por el ojo humano (clasificación A), mientras que una diferencia mayor de 3 unidades sí es perceptible (clasificación B) de acuerdo a la clasificación propuesta por Quenard (17). $a *_{2}, b_{2}{ }_{2}$ y $L^{*}{ }_{2}$ are the values of the colour coordinates ( $a^{*}$ and $b^{*}$ ) and of the lightness $\left(L^{*}\right)$ after the application of anti-graffiti.

According to the classification of $\Delta E^{*} a, b$ values proposed by Quenard (17), the results of this parameter were evaluated establishing the limit of 4 units of colour for the changes which are not perceptible by the human eye (classification A). However, when $\Delta E^{*}{ }_{a, b}$ was higher than 4 units, it was considered that the colour change between the untreated sample and treated sample was perceptible (classification B).

The results indicate that the darkest substrates are the brick and the Belgian blue limestone (L: 50.87 y 55.44 respectively), whereas the lightest one is the Roman travertine (L: 81.89). In this sense, in most of the cases the application of anti-graffiti darkens the substrate $\left(\Delta L^{*}\right.$ $<0)$, being in general anti-graffiti 3 (polyurethane) and 4 (ethyl methacrylate) the products most darkening the substrates. This can be due to a higher amount of product absorbed by the substrates, as previously discussed, in the case of anti-graffiti 3 and 4. The total colour differences $\left(\Delta E^{*} a, b\right)$ are perceptible in all substrates after the application of these two products, where the substrates most affected the calcareous sandstones in the case of anti-graffiti 3 and the Belgian blue limestones in the case of anti-graffiti 4. However, the paraffin-based anti-graffiti (anti-graffiti 2) presents a total colour difference higher than 4 units only in the Belgian blue limestone, the Galdakao siliceous sandstone and the brick, being the lightness the parameter varying most in the three cases. Nevertheless, it is worth highlighting that except in the case of the Belgian blue limestone, the total colour differences are not significant. Finally, the antigraffiti 1 (acrylate copolymer) only produces nonperceptible colour changes in one substrates from the 8 studied materials, and this is the Villamayor siliceous sandstone, but in general, the values of $\Delta E^{*} a, b$ are lower in comparison to those of anti-graffiti 3 and 4.

With regard to the changes in gloss conferred by the application of anti-graffiti, Table 8 shows the differences between the measurements performed before and after the application of each anti-graffiti:

For the evaluation of gloss, it was considered that a difference lower than 3 units with respect to the untreated sample is not perceptible by the human eye (classification A), whereas a difference higher than 3 units is perceptible (classification $B$ ) following the classification proposed by Quenard (17). 
Tabla 8 / Table 8

Resultados de las medidas de brillo.

Results of the measurements of gloss.

\begin{tabular}{|c|c|c|c|c|c|c|c|c|c|c|}
\hline \multirow{2}{*}{$\begin{array}{c}\text { Propiedad / } \\
\text { Property }\end{array}$} & \multirow[b]{2}{*}{$\begin{array}{l}\text { Sustrato / } \\
\text { Substrate }\end{array}$} & \multirow{2}{*}{$\begin{array}{c}\begin{array}{c}\text { Patrón / } \\
\text { Blank }\end{array} \\
\text { Valor } \\
\text { medio I } \\
\text { Average } \\
\text { value }\end{array}$} & \multicolumn{2}{|c|}{ AG-1 } & \multicolumn{2}{|c|}{ AG-2 } & \multicolumn{2}{|c|}{ AG-3 } & \multicolumn{2}{|c|}{ AG-4 } \\
\hline & & & $\begin{array}{c}\text { Valor } \\
\text { medio / } \\
\text { Average } \\
\text { value }\end{array}$ & $\begin{array}{l}\text { Clas. I } \\
\text { Class. }\end{array}$ & $\begin{array}{c}\text { Valor } \\
\text { medio / } \\
\text { Average } \\
\text { value }\end{array}$ & $\begin{array}{l}\text { Clas. I } \\
\text { Class. }\end{array}$ & $\begin{array}{c}\text { Valor } \\
\text { medio / } \\
\text { Average } \\
\text { value }\end{array}$ & $\begin{array}{l}\text { Clas. I } \\
\text { Class. }\end{array}$ & $\begin{array}{c}\text { Valor } \\
\text { medio / } \\
\text { Average } \\
\text { value }\end{array}$ & $\begin{array}{l}\text { Clas. I } \\
\text { Class. }\end{array}$ \\
\hline \multirow{8}{*}{$\begin{array}{c}\text { Brillo / Gloss } \\
\text { (diferencia en } \\
\text { unidades } \\
\text { de brillo / } \\
\text { difference in } \\
\text { gloss units) }\end{array}$} & VILLA & 0.2 & 0.8 & A & 0.6 & A & 0.8 & A & 0.7 & A \\
\hline & GALD & 0.2 & 0.8 & A & 0.6 & A & 0.8 & A & 1.1 & A \\
\hline & STRT & 0.3 & 1.1 & A & 1.6 & A & 1.4 & A & 1.1 & A \\
\hline & BAUM & 0.2 & 1.1 & A & 3.8 & B & 1.6 & A & 6.5 & B \\
\hline & BLUE & 1.6 & 3.6 & B & 4.1 & B & 1.9 & A & 51.8 & B \\
\hline & BALE & 0.6 & 1.7 & A & 2.0 & A & 1.7 & A & 7.2 & B \\
\hline & TRAV & 3.0 & 5.9 & B & 6.5 & B & 2.2 & A & 31.3 & B \\
\hline & LADR & 0.2 & 0.7 & A & 0.9 & A & 0.8 & A & 0.6 & A \\
\hline
\end{tabular}

Considerando esto, se observó que todos los anti-grafiti comerciales son aceptables (clasificación A) en el caso de las areniscas silíceas de Villamayor y Galdakao, arenisca calcárea de Strtenica y en el caso del ladrillo. Estos sustratos presentan valores de porosidad altos y similares $(15,1 \% ; 15,8 \% ; 15,8 \%$ y $19,5 \%$, respectivamente) y tamaño de poro grande $(2,4 \mu \mathrm{m} ; 1,3 \mu \mathrm{m} ; 1,7 \mu \mathrm{m}$ y 3,1 $\mu \mathrm{m}$, respectivamente). Teniendo en cuenta que el brillo especular es proporcional a la reflectancia de la superficie, la cual es dependiente a su vez de la rugosidad de la misma (18), es decir, a mayor rugosidad superficial, mayor brillo, puede deducirse que la aplicación de antigrafiti en estos sustratos no produce cambios significativos en la rugosidad de estos materiales. En cambio, la aplicación de anti-grafiti en sustratos de baja porosidad y tamaño de poro pequeño, produce cambios de brillo perceptibles en el caso de los anti-grafiti 4 (metacrilato de etilo), anti-grafiti 2 (polímeros parafínicos) y anti-grafiti 1 (copolímero de acrilato), lo que sugiere que el producto aplicado forma una capa de mayor diferencia en su rugosidad, en comparación al sustrato no tratado. El anti-grafiti 3 (poliuretano) no produce cambios en el brillo perceptibles por el ojo humano.

En conclusión, puede decirse que de los 4 anti-grafiti comerciales analizados, el producto con base parafínica es el que menores cambios de color produce en los sustratos y el anti-grafiti de base poliuretano, el que menores cambios de brillo produce.

\section{CONCLUSIONES}

El comportamiento de los tratamientos anti-grafiti depende, en gran medida, no sólo de las características del producto anti-grafiti, sino también del tipo de sustrato sobre el que se aplica. Es por ello que resulta fundamental efectuar ensayos de idoneidad como las medidas
Considering this, it was observed that all commercial anti-graffiti are acceptable (classification A) in the case of the Villamayor and Galdakao siliceous sandstones, Strtenica calcareous sandstone and in the case of the brick. These substrates present high and similar porosity values (15.1\%; 15.8\%; $15.8 \%$ y $19.5 \%$ respectively) and big pore size $(2.4 \mu \mathrm{m} ; 1.3 \mu \mathrm{m} ; 1.7 \mu \mathrm{m}$ y $3.1 \mu \mathrm{m}$ respectively). If it is considered that specular gloss is proportional to surface reflectance, which is dependent on the roughness of this surface (18) i.e. the higher the roughness, the higher the gloss, it can be deduced that the application of anti-graffiti in these substrates does not produce any significant change in the roughness of these materials. However, the application of anti-graffiti in low porosity and small pore size substrates leads to perceptible gloss changes in the case of anti-graffiti 4 (ethyl methacrylate), anti-graffiti 2 (paraffin polymers) and anti-graffiti 1 (acrylate copolymer), which suggests that the applied product forms a layer of a greater different in its roughness in comparison to the untreated substrate. Anti-graffiti 3 (polyurethane) does not produce gloss changes which can be perceived by the human eye.

In conclusion, it can be said that from the 4 tested commercial anti-graffiti, the paraffin based product is that producing the least colour changes in the substrates and the polyurethane based anti-graffiti the anti-graffiti producing the least gloss changes.

\section{CONCLUSIONS}

The behaviour of the anti-graffiti treatments depends to a large extent not only on the characteristics of the antigraffiti product, but also on the type of substrate where it is applied. For this reason it is essential to perform suitability tests such as measurements of the hydric 
de las propiedades hídricas (absorción de agua a presión atmosférica, succión capilar, permeabilidad al vapor de agua, comportamiento frente al secado) previamente a la aplicación de estos productos sobre sustratos porosos. En el caso de los materiales históricos, los cambios de color y brillo que el anti-grafiti puede conferir al sustrato poroso también deben de tenerse en cuenta.

La aplicación de anti-grafiti sobre un sustrato poroso reduce las propiedades hídricas del material, es decir, la transferencia libre de agua tanto en fase líquida como en fase vapor se ve impedida debido al efecto barrera que el recubrimiento confiere al sustrato.

De todos los anti-grafiti ensayados, la emulsión acuosa de polímeros parafínicos y el copolímero de acrilato son los productos que en menor medida reducen los valores de las propiedades hídricas; el anti-grafiti de base parafínica presenta menores cambios de color, por lo que podría decirse que es más idóneo en la protección de sustratos porosos del Patrimonio Histórico, aunque debe tenerse en cuenta que en los sustratos menos porosos puede producir cambios de brillo perceptibles por el ojo humano.

El etil metacrilato y el poliuretano (especialmente, este último) forman una película casi impermeable sobre el sustrato poroso que dificulta significativamente el intercambio libre de agua tanto en fase vapor como en fase líquida. Además, producen cambios de color perceptibles por el ojo humano, aunque debe mencionarse que el brillo, en el caso del poliuretano, no se ve afectado.

\section{AGRADECIMIENTOS}

Los autores desearían agradecer la ayuda de la Comisión Europea (contrato no: 513718) y el Ministerio de Educación y Ciencia (MAT2005-25563-E). properties (water absorption at atmospheric pressure, capillary suction, water vapour permeability, drying behaviour) prior to performing the application of such products on porous substrates. In the case of historical materials, the colour and gloss changes that the antigraffiti could confer to the substrate must also be considered.

The application of anti-graffiti on a porous substrate reduces the hydric properties of the material i.e. the free water exchange both in the vapour phase and in the liquid phase is hindered due to the barrier effect that the coating confers to the substrate.

Among all the tested anti-graffiti, the aqueous emulsion of paraffinic polymers and the acrylate copolymer are the products reducing to a lesser extent the values of the hydric properties; the paraffin based anti-graffiti presents lower colour changes, thus it could be said that it is more suitable for the protection of the porous substrates of Cultural Heritage. However, it must be borne in mind that it can produce perceptible colour changes in the least porous substrates.

The ethyl methacrylate and the polyurethane (especially the latter) form an almost impermeable coating on the porous substrate which hinders significantly the free water exchange both in the vapour phase and in the liquid phase. Additionally, they produce colour changes which can be perceived by the human eye, although it must be mentioned that the gloss, in the case of the polyurethane, is not affected.

\section{ACKNOWLEDGEMENTS}

This research was funded by the European Commission (contract $n^{\circ}:$ 513718) and the Spanish Ministry of Education and Science (MAT2005-25563-E).

\section{BIBLIOGRAFÍA / BIBLIOGRAPHY}

(1) Castleman, C.: "Getting Up, Subway Graffiti in New York". The MIT Press, Massachusetts (1982).

(2) Weaver, M. E.: "Conserving Buildings: A Guide to Techniques and Materials". John Wiley \& Sons, Inc. (1993).

(3) Allison, M.: "Use of fluoropolymers for the protection of masonry and other materials used in the construction industry". Proceedings of the $2^{\text {nd }}$ International Fluorine in the Service of Man Conference, "Fluorine in coatings", paper 27 (1994), p. 2.

(4) Zielecka, M.: "Anti-graffiti protection of porous building materials". Conference Proceedings XXV FATIPEC Congress, vol. 2 (2000), pp. 311-321.

(5) Temtchenko, M.; Novelli, S.; Delucchi, M.; Cerisola G.: "New fluorinated protective coatings", Pitture e Vernici Europe, vol. 77 (2001), pp. $15-20$.

(6) Janssen, J. R.; Everaerts, A. I.; O'Keefe, D. R.; Sheffield, W. F.: "Graffiti and/or environmental protective film stack article having removable sheets, film protected substrates, and use", 3m Innovative Properties Company, PTC Int. Appl. (2000), p. 54.

(7) Ashurst, N.; Chapman, S.; MacDonald, S.; Butlin, R.; Murry, M.: "An investigation of sacrificial graffiti barriers for historic masonry", English Heritage Research Transactions, vol. 2: Stone (2002), James and James, London (2002). 
(8) Borchard-Tuch, C.: "Beschitungen contra Graffiti", Chemie in unserer Zeit, vol. 39 (2005), pp. 355-357. doi:10.1002/ciuz.200590068

(9) Wood, R.: "The writings on the wall", Materials World (August 2004), pp. 30-32.

(10) Villegas Sánchez, R.: "Metodología para la evaluación y estudio previo de tratamientos", Metodología de diagnóstico y evaluación para la conservación de los edificios históricos, Cuadernos Técnicos (2003), pp. 194-207.

(11) Navarro Gascón, J. V.: "Tratamientos sobre piedra: requisitos que condicionan su viabilidad", I Jornadas de Caracterización y Restauración de Materiales Pétreos en Arquitectura, Escultura y Restauración, tomo II (2001), pp. 109-115.

(12) Esbert, R. M.; Ordaz, J.; Alonso, F. J.; Montoto, M.: Manual de diagnosis y tratamiento de materiales pétreos y cerámicos. Col·legi d'Aparelladors i Arquitectes Tècnics de Barcelona. Barcelona (1997).

(13) Lubelli, B. B.; van Hees, R. P. J.; van de Weert, T. G.: "The drying behaviour of building materials treated with anti-graffiti", Proceedings of Hydrophobe V - Fifth International Conference on Water Repellent Treatment of Building Materials, Brussels (2008), pp. 85-94.

(14) De la Torre López, M. J.: "Caracterización de las propiedades hídricas de los materiales lapídeos", Metodología de diagnóstico y evaluación para la conservación de los edificios históricos, Cuadernos Técnicos (2003), pp. 104-111.

(15) Urquhart, D.: "The treatment of graffiti on historic surfaces - Advice on graffiti removal procedures, anti-graffiti coatings and alternative strategies". Historic Scotland. Edinburgh (1999).

(16) Wedler, E.; Charola, A. E.: "Water and its interaction with porous inorganic building materials", Proceedings of Hydrophobe $V-$ Fifth International Conference on Water Repellent Treatment of Building Materials, Brussels (2008), pp. 57-73.

(17) Quenard, D.: "Water repellent treatments test methods and performance criteria", Final Technical Report (2002).

(18) Meeten, G. H.: Optical Properties of Polymers. Elsevier Applied Science, London (1986).

\section{NORMAS / STANDARDS}

EN 13755: 2002, "Métodos de ensayo para piedra natural - Determinación de la absorción de agua a presión atmosférica"/ "Natural stone test methods - Determination of water absorption at atmospheric pressure".

EN 1925: 1999, "Métodos de ensayo para piedra natural - Determinación del coeficiente de absorción de agua por capilaridad" / "Natural stone tests method - Determination of water absorption coefficient capillarity".

EN ISO 2813: 1999, "Pinturas y barnices - Determinación del brillo especular de películas de pintura no metálicas a $20^{\circ}, 60^{\circ}$ y $85^{\circ}$ " / "Paints and varnishes - Determination of specular gloss of non-metallic paint films at 20 degrees, 60 degrees and 85 degrees".

EN ISO 7783-2: 1999, "Pinturas y barnices - Materiales de recubrimiento y sistemas de recubrimiento para albañilería exterior y hormigón - Parte 2: Determinación y clasificación de la velocidad de transmisión agua-vapor (permeabilidad)" / "Paints and varnishes - Coating materials and coating systems for exterior masonry and concrete - Part 2: Determination and classification of water vapour transmission rate (permeability)".

ISO 7724-2: 1994, "Pinturas y barnices - Colorimetría - Parte 2: Medida del color" / "Paints and varnishes - Colorimetry - Part 2: Colour measurement".

ISO 7724-3: 1994, "Pinturas y barnices - Colorimetría - Parte 2: Cálculo de diferencias de color" / "Paints and varnishes - Colorimetry - Part 3: Calculation of colour differences".

RILEM 25-PEM, Protection et érosion des monuments: 1980, Test No. II.4, "Evaporation curve". 